\title{
The role of membrane- inner retina adherence in predicting simultaneous internal limiting membrane peeling during idiopathic epiretinal membrane surgery
}

${ }^{1}$ Ophthalmica Eye Institute, Thessaloniki, Greece

${ }^{2}$ Moorfields Eye Hospital, London, UK

${ }^{3}$ Attikon University Hospital, 2nd Department of Ophthalmology, University of Athens Medical School, Athens, Greece

Correspondence:

P Stavrakas, Attikon University Hospital, 2nd

Department of

Ophthalmology, University of Athens Medical School, 1 Rimini Street, 12462 Athens, Greece

Tel: +30 6944276610 ; Fax: +302109811138 E-mail: panos.stavrakas@ yahoo.com

Received: 6 April 2016 Accepted in revised form: 7 November 2016

Published online:

23 December 2016

\begin{abstract}
Purpose To correlate the frequency and extent of simultaneous inadvertent internal limiting membrane (ILM) peeling during idiopathic epiretinal membrane (ERM) removal with characteristics of ERM adherence demonstrated on pre-operative spectral domain optical coherence tomography (SD-OCT).

Patients and methods This is a prospective, observational, case series of patients undergoing pars plana vitrectomy for idiopathic ERM. Inner retina-ERM adhesion was categorized as focal, broad or complete in five anatomic locations at macular area based on preoperative SD-OCT findings. The extent of spontaneous ILM peeling was quantified on a scale $\mathbf{0 - 1 0 0 \%}$ in each of the aforementioned anatomic locations by the operating surgeons who were masked to the OCT characteristics. All operations were recorded with a high definition recording system and the area of simultaneous ILM peel was quantified by a second masked observer. The final extent of spontaneous ILM peel was calculated as the average of the two scores.

Results Thirty consecutive subjects who underwent surgery for idiopathic ERM were included in the study. Evidence of simultaneous ILM peeling was identified in $80.3 \%$ of individuals. With regards to the type of ERM-macula adhesion, inadvertent ILM peel was observed in $70 \%$ of the patients who pre-operatively showed complete adhesion, in $43 \%$ with broad adhesion and in only $21 \%$ with focal adhesion $(P<0.001)$. The extent of the spontaneous ILM peel during removal of ERM was also significantly
\end{abstract}

P Tranos ${ }^{1}$, L Wickham², N Dervenis', A Vakalis', $S$ Asteriades ${ }^{1}$ and P Stavrakas ${ }^{3}$ dependent on the type of ERM-inner retina adhesion. Total simultaneous ILM peel was observed in $59 \%$ of locations with complete ERM-macula adhesion but only in $22 \%$ and $7 \%$ of locations with broad and focal adhesion respectively $(P<0.001)$. Conclusions Simultaneous ILM peel is a frequent occurrence during ERM surgery, especially when there is complete or broad ERM adherence to the macula. The type of ERM-inner retina adhesion represents a valid predictor of the extent of simultaneous ILM peel during removal of ERM. Thorough evaluation of preoperative OCT may be a useful tool in determining a safer, more simplistic strategy in ERM surgery.

Eye (2017) 31, 636-642; doi:10.1038/eye.2016.285; published online 23 December 2016

\section{Introduction}

Epiretinal membrane (ERM) consists of a semitranslucent, avascular, fibrocellular membrane that proliferates on the inner surface of the retina and produces various degrees of visual impairment. Machemer et al were the first to describe pars plana vitrectomy (PPV) with ERM peeling for visually significant ERMs. ${ }^{1}$ Satisfactory functional and structural outcomes have been consistently reported for PPV and ERM removal thereafter. ${ }^{2-4}$

The internal limiting membrane (ILM) can act as a scaffold for cellular proliferation in the pathophysiology of disorders affecting the vitreomacular interface. ${ }^{5}$ Peeling of the ILM has been proposed as a method to ensure complete removal of ERM, improve the prognosis of surgical treatment and reduce the risk of ERM 
recurrence. ${ }^{6}$ However, current literature has failed to provide strong evidence for the beneficial role of ILM removal during ERM surgery, with most studies being retrospective and divergent regarding their outcomes. ${ }^{7-15}$ Moreover, there are concerns about the safety of ILM peeling and the risk of potential mechanical or toxic trauma to the inner and outer retina during the procedure. ${ }^{12,15}$ Interestingly, several studies have shown that ILM peel may occur unintentionally during removal of ERM. Histological analysis of ERM specimens has provided evidence that segments of ILM are commonly present in the ERMs that have been removed, ${ }^{7}$ whereas other clinical studies using vital dyes following ERM peeling reported that ILM is frequently still intact on the inner retinal surface. ${ }^{16}$ Although the inadvertent ILM peel during ERM surgery has been well documented, there is no published data so far, to associate this occurrence with preoperative patients' characteristics. Correlating baseline optical coherence tomography (OCT) features to the frequency of intraoperative spontaneous ILM peel would provide a useful predictive tool facilitating effective surgical planning.

The introduction of modern spectral domain OCT (SDOCT) instruments has allowed the identification of anatomical changes that occur in the macular architecture before and after ERM surgery. ${ }^{12,17-22}$ Further, it has facilitated detailed visualization of the membranes and their relationship to the retinal surface. The type of ERMmacula adhesion has been shown to play a role in different aspects of ERM surgery including surgical difficulty. ${ }^{23}$ It is therefore possible that the extent of simultaneous unintentional ILM peeling during removal of ERM may also relate to OCT characteristics.

In this study, we evaluated the frequency and extent of simultaneous ILM peeling during removal of idiopathic ERM and correlated it with different types of ERM-retina adhesion based on preoperative SD-OCT images.

\section{Materials and methods}

Thirty-eight consecutive patients undergoing PPV for idiopathic ERM removal at the Ophthalmica Eye Institute, Thessaloniki, Greece, were screened for participation in the prospective study. All eligible patients presented with idiopathic ERM, clinically significant metamorphopsia confirmed on Amsler grid chart and visual acuity of the affected eye $<6 / 9$. Individuals with a history of previous vitreoretinal surgery, secondary ERM (eg, due to diabetic maculopathy, retinal vascular disorders, retinal detachment, age-related macular degeneration, ocular trauma, or uveitis), recurrent ERM, or ERM associated with lamellar macular hole were excluded from the study.

Institutional review board/ethics committee approval was obtained before the study commenced.

\section{OCT imaging and epiretinal membrane-macula adhesion classification}

All subjects underwent a complete baseline clinical assessment within 1 month before surgery, including Snellen best corrected visual acuity measurement, dilated fundus examination, and OCT, using the Spectralis SDOCT (Heidelberg Engineering, Vista, CA, USA). The ERM-macula adhesion classification was based on the methodology proposed by Kim et al. ${ }^{23}$ Briefly, a raster of vertical and horizontal SD-OCT images of the ERM structure was obtained over the macula. With the aid of an ETDRS overlay, the posterior pole was divided into five anatomic locations including a foveal (central 1000 microns) and four macular quadrants (superior, inferior, nasal, and temporal) extending up to the large temporal vascular arcades. Calipers were then used to measure the length of ERM-retinal adherence and subsequently divided by the total length of ERM to obtain ERM-retinal adhesion percentage. The above was repeated for each

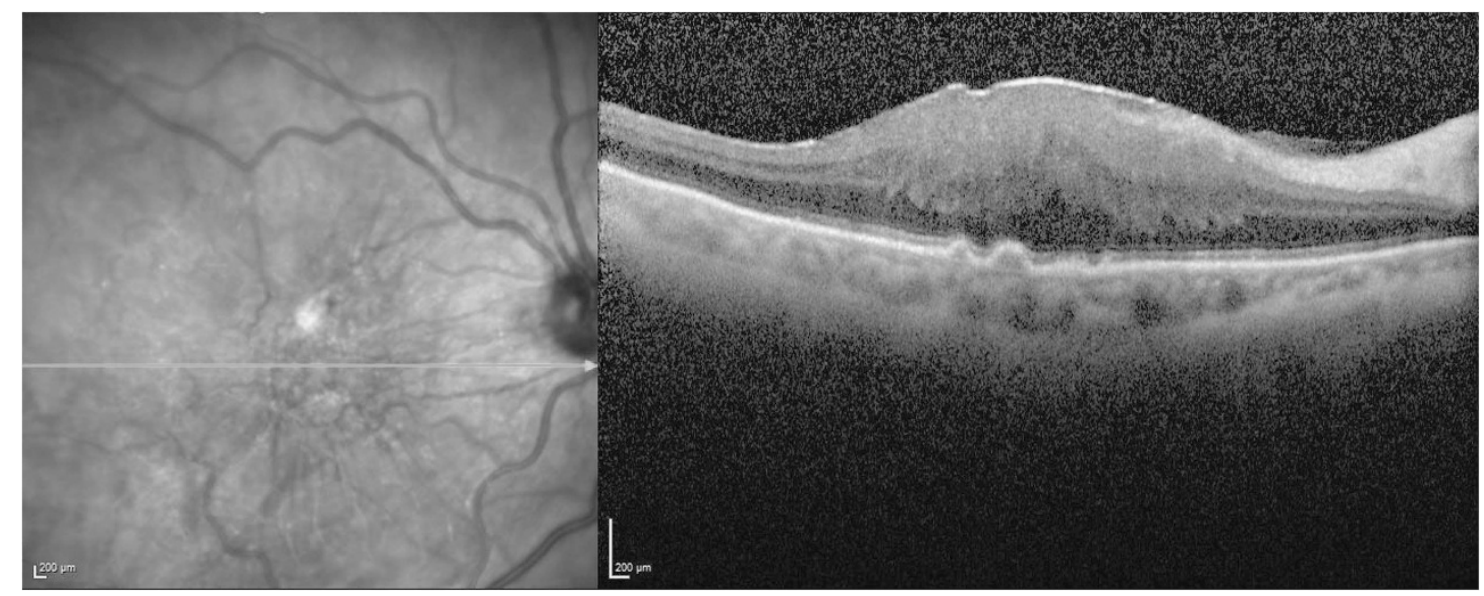

Figure 1 Focal $(<50 \%)$ ERM-retinal adherence is seen in both nasal and temporal to the fovea along the horizontal axis using SD-OCT. ERM-retinal adherence is broad (50-90\%) at the center of the fovea on the same section. 
vertical and horizontal meridian and subsequently results were averaged to obtain the final percentage of ERMinner retina adherence in each anatomic location. All calculations were performed in a masked fashion by two observers.

The extent of ERM-retinal adhesion was then categorized as focal, broad, or complete. ${ }^{23}$ Focal adhesion was defined as ERM-retinal adhesion $<50 \%$; broad adhesion between 50 and $90 \%$; and complete adhesion $>90 \%$ (Figures 1 and 2). In cases where the classification did not agree between the observers for any of the anatomic locations, the type of the ERM adherence to the retina was determined by a third masked observer.

\section{Surgical procedure and simultaneous ILM peel quantification}

Following informed consent for the study, patients were listed for PPV and ERM peel. All subjects underwent a three-port 23 or 25 gauge PPV by three experienced retinal surgeons (PT, $\mathrm{AV}$, and $\mathrm{SA})$. Intravitreal triamcinolone to verify that the posterior hyaloid had been removed was used only when required. Trypan blue (membrane blue $0.15 \%$, DORC, Zuidland, The

Netherlands) was injected into the vitreous to stain the ERM and washed out immediately. The ERM was then peeled and Brilliant blue G (Brilliant Peel, Fluoron, Geuder, Germany) was used to stain the ILM. The presence of residual ILM at the area of the pre-existing ERM was recorded in five different anatomic locations including the central 1000 microns and the four macular quadrants described above and quantified by the surgeon on a scale $0-100 \%$ for each anatomic location $(100 \%$ meaning total simultaneous ILM peel during ERM removal). Subsequently, ILM peel was initiated peripheral to the location of the pre-existing ERM and directed toward the fovea, confirming the absence or presence of ILM. In order to increase study reliability and avoid bias, all operations were recorded with a Zeiss (Carl Zeiss Meditec AG, Jena, Germany) high-definition three-chip recording system and the area of simultaneous ILM peel was quantified by a second masked observer who was also an experienced vitreoretinal surgeon. The final extent of simultaneous ILM peel was the average of the intraoperative and postoperative measurements documented by the operating surgeon and the video observer, respectively.

Primary outcome measure was the extent of simultaneous ILM peeling in patients undergoing PPV for idiopathic ERM and its frequency in relation to different types of ERM-retina adhesion based on preoperative SDOCT images. Secondary outcome measure was the effect of patients' characteristics or other intraoperative parameters on the occurrence of simultaneous, inadvertent ILM peeling in ERM surgery.

Statistical analysis was performed with SPSS software for Windows (version 16.0 SPSS, Inc, Chicago, IL, USA). The distribution of all variables was examined using frequency tables and descriptive statistics. Box-andwhisker plots and histograms were used to display the different data distributions. Parametric methods, including independent Student's $t$-test, ANOVA, and linear regression, were used for normally distributed variables. Non-parametric tests such as Mann-Whitney $U$ and Wilcoxon signed rank tests were used for variables that did not show normality. The relationship of the

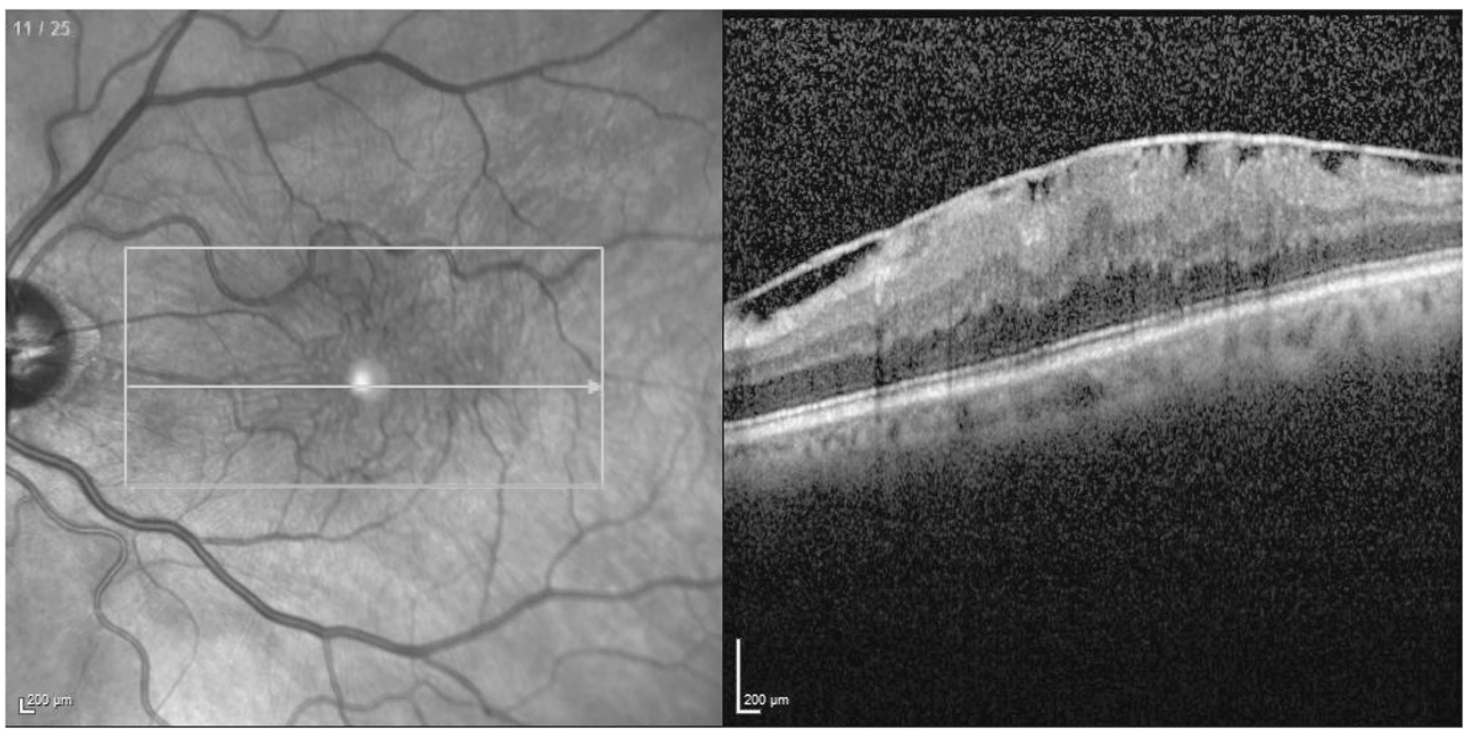

Figure 2 Complete (90\%) ERM adherence to the inner retina along the posterior pole (both nasal and temporal to the fovea as well as at the center of the fovea). 
simultaneous ILM peel with patients' demographics or intraoperative variables was examined using the Spearman and Pearson rank correlation test. The relationship between categorical variables was also evaluated using the $\chi^{2}$-test. All tests of association were considered to be statistically significant if $P<0.05$.

The study was conducted according to the tenets of the Declaration of Helsinki.

\section{Results}

Thirty eyes of 30 consecutive patients who matched the inclusion criteria were included in the study and eight eyes were excluded. One patient was ultimately excluded from the statistical analysis as both surgeon and observer of the operating video failed to provide a definite grading of unintentional ILM peeling. The male to female ratio in the 29 patients was $14: 15$ and their mean age was $70 \pm$ 7 years. ERM-retinal adherence pattern resulted in complete concordance $(\kappa=1)$ in each quadrant between the horizontal and vertical raster scans for each observer. Of the 29 subjects (145 anatomic locations) that were included in the data analysis, assessment of the ERMretinal adherence measurements for interobserver agreement, resulted in 95.8\% (139/145) concordance between masked observers.

Evidence of simultaneous ILM peel during removal of ERM was observed in 23 eyes (80.3\%), whereas in six eyes (19.7\%) ILM remained intact following ERM peeling. In nine eyes (31\%) there was total-100\%—-simultaneous

Table 1 Data summary of recruits (29 eyes of 29 patients)

\begin{tabular}{ll}
\hline Sex & \\
Male & 14 \\
Female & 15 \\
Age & \\
$\quad$ Range & $60-78$ \\
$\quad$ Mean (SD) & $70(7)$ \\
& \\
LogMAR baseline visual acuity & $0.35-0.9$ \\
Range & $0.6( \pm 0.2)$ \\
Mean (range) & \\
& \\
Size of epiretinal membrane (disc areas) & $5-35$ \\
Range & $12(9)$ \\
Mean (range) & \\
Size of sclerotomies & 8 \\
23 gauze & 21 \\
25 gauze & \\
Extent of inadvertent ILM peel (percentage) & $19.7 \%$ \\
$0 \%$ & $29.6 \%$ \\
$1-50 \%$ & $19.7 \%$ \\
$51-90 \%$ & $31.0 \%$ \\
$100 \%$ & \\
\hline
\end{tabular}

ILM peel, and in 14 eyes (48.3\%) simultaneous ILM peel was only partial (Table 1 ).

With regards to the type of ERM-macula adhesion, simultaneous ILM peel was observed in $70 \%$ of the locations that pre-operatively showed complete adhesion, in $43 \%$ of the locations with broad adhesion and in only $21 \%$ of the locations with focal adhesion. This difference was statistically significant between the three groups $(P<0.001)$ (Figure 3).

The extent of the spontaneous ILM peel during removal of ERM was also significantly dependent $(P<0.001)$ on the type of ERM-inner retina adhesion. Total simultaneous ILM peel was observed in 59\% of anatomic locations with complete ERM-macula adhesion but only in $22 \%$ and $7 \%$ of locations with broad and focal adhesion, respectively. Conversely, ILM remained intact following removal of ERM in $59 \%, 45 \%$, and $16 \%$ of the

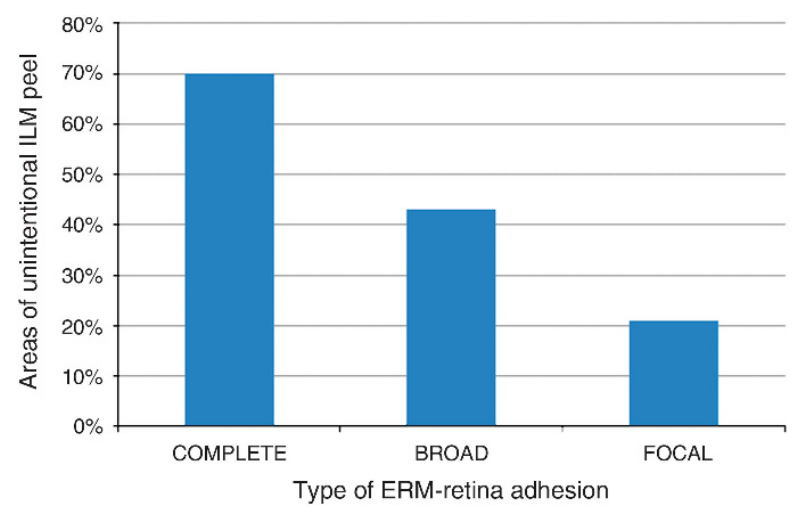

Figure 3 Percentage of anatomic locations with simultaneous ILM peel with regards to the type of ERM-macula adhesion.

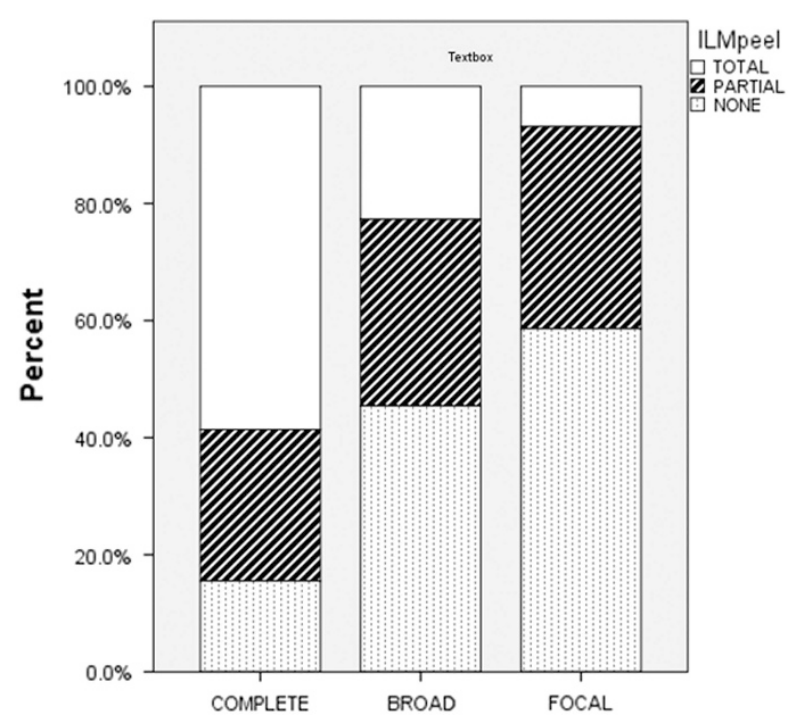

Figure 4 Extent of simultaneous ILM peel with regards to the type of ERM-retina adhesion. 
focal, broad, and complete ERM-macula adhesion, respectively (Figure 4).

Further analysis revealed that spontaneous ILM peel during ERM surgery was independent of patients' demographics, baseline visual acuity, size of ERM, or intraoperative parameters including size of sclerotomies and duration of surgery $(P>0.05$, Mann-Whitney $U$ test).

\section{Discussion}

With the advent of high-resolution SD-OCT instruments, a wealth of information on vitreoretinal interface has become available. ${ }^{19,21-23}$ Better definition of the geometry of ERM adhesion to the inner retina has revealed different types of adherence including focal, broad, or complete. ${ }^{23}$ These adhesion patterns have been shown to correlate with varying degrees of surgical difficulty during ERM removal. ${ }^{23}$ Moreover, en face OCT reveals elevated areas of ERM, facilitating initial grasping of the membrane and potentially predicting visual outcomes after surgery. ${ }^{24}$ Therefore, meticulous analysis of preoperative OCT is becoming increasingly important for effective surgical planning to improve visual and anatomical outcomes of ERM surgery.

Partial or total inadvertent ILM peel during ERM removal has been reported in $40-70 \%$ of cases. ${ }^{7,9,25-27}$ Oh $e$ e $a l^{9}$ in a series of 20 eyes observed that simultaneous ILM peel occurred in $60 \%$ of the operated eyes, whereas in other studies the corresponding incidence was $\sim 40 \%$. Interestingly, the Pan-American Collaborative Study Group documented that in all 74 eyes that underwent ERM peeling, the ILM was always present on the macular surface either damaged or undamaged. ${ }^{16}$ Other research groups have used light ${ }^{26}$ or electron microscopy ${ }^{7}$ to analyze the ERM specimens. Bovey et $a l^{7}$ confirmed the presence of long segments of ILM in $74 \%$ of the peeled ERMs, but did not quantify the simultaneously removed ILMs during ERM surgery.

Our clinical study specifically addressed the issue of spontaneous ILM peel during ERM surgery and prospectively evaluated its incidence in relation to preoperative OCT characteristics. The results presented herein showed clearly that unintentional ILM peel during ERM removal occurs in the vast majority of cases and approximately in one-third of those eyes simultaneous peel involves the entire ILM underlying the ERM.

The incidence of $80 \%$ partial or total inadvertent ILM peel found in our series is higher compared with previous reports. This is probably attributable to the strict study protocol ensuring accurate evaluation of spontaneous ILM peel, as well as to exclusion of secondary ERMs, which are known to be more focally adherent to the retinal surface. Bovey et al implied in their study that simultaneous removal of the ILM during ERM peeling was more common in secondary ERMs. The high rate of spontaneous ILM peeling found in our series of idiopathic ERMs seems to support their observations.

Interestingly, the extent of simultaneous ILM peel is significantly dependent on the type of ERM adhesion to the inner retina. In areas where ERM was greater than $90 \%$ adherent to the retina, total simultaneous ILM removal was over eight times more likely to occur, as opposed to areas where ERM was only focally attached, with $<10 \%$ adhesion to the retina.

Conversely, less extensive ERM adherence to the inner retina made it more likely to maintain the ILM intact with no fragmentation of the latter during ERM peel. Therefore, the extent of ERM-retina adhesion is a significant predictive factor for inadvertent ILM peel in ERM surgery and can facilitate effective surgical planning.

Although the role of ILM peeling following removal of ERM remains controversial, there is a rising number of vitreoretinal surgeons who favor this adjunct procedure. Recent surveys have revealed that the number of those routinely performing ILM peeling escalated from $25 \%$ in 2008 to $44 \%$ in $2010 .{ }^{10}$ However, the potential benefit or possible deleterious effects of this additional surgical maneuver is still to be determined. Previous comparative studies evaluating the effect of ILM peeling on final visual outcomes, re-proliferation rate of the epimacular membrane and safety of the procedure, often provided contradictory results. ${ }^{7-15}$

Removal of ILM is known to cause mechanical trauma to the retinal nerve fiber layer (RNFL) but the observed changes seem to be subclinical. ${ }^{25-29}$ Early transient swelling of the arcuate RNFL ${ }^{30}$ and dissociated optic nerve fiber layer ${ }^{31-33}$ have been reported to occur after ILM peeling but they do not appear to influence the visual recovery or to cause reduced retinal sensitivity. ${ }^{30-34}$ However, it has been postulated that ILM peeling may be related to selective superotemporal, temporal, and inferotemporal RNFL thickness reduction and retinal weakening caused by Müller cell damage, which can result in paracentral hole formation. ${ }^{35}$

Although not entirely proven it seems that trauma to RNFL and Müller cell following ILM peeling is attributable to inadvertent surgical injury, absence of ILM, and potential toxicity of vital stains used during the procedure. $10,13,15,24$ Avoiding any of the above insults could possibly improve the safety profile of ERM surgery resulting in better outcomes. Consequently, the optimum surgical strategy would combine best functional and anatomical results with minimum intervention, averting unnecessary surgical maneuvers on the surface of the macula. A double stain technique aiming at the complete removal of the ILM following ERM peel, would potentially result in additional mechanical trauma from the peeling forceps and the exposure time to the endoillumination light. Recent reports have shown that postoperative visual acuity is significantly correlated to the 
number of grasping attempts ${ }^{24}$ and if this could be avoided by predicting simultaneous ERM/ILM peel, surgical outcomes could be improved. In cases that ILM peel is considered necessary, recent technological advances including intraoperative OCT and vital dyes which stain both ERM and ILM may also enhance safety and efficacy of ERM surgery. ${ }^{36}$

We appreciate certain limitations of our study including the lack of histopathological analysis of the specimens and restriction of the sample to only idiopathic ERMs. Despite the absence of laboratory verification of our results, detailed clinical documentation and quantification of the spontaneous ILM peel, facilitated by vital dyes guaranteed reliability. Moreover, results were re-evaluated by masked observers who carefully studied the operating video to confirm the surgeons' intraoperative clinical impression. By restricting our study to idiopathic membranes, the study did not provide any information on simultaneous ILM peel associated with secondary ERM surgery, however, this assured a homogenous sample minimizing bias. Previous reports supported that secondary ERMs are more focally adherent to the retinal surface, a characteristic which could be a confounding factor in our statistical analysis.

In summary, simultaneous ILM peel is a frequent occurrence during ERM surgery, especially when there is broad or complete ERM-macula adhesion on OCT. We report for the first time that the extent of ERM-inner retina adhesion represents a valid predictor of the simultaneous ILM peel during removal of ERM. Thorough evaluation of preoperative OCT may be a useful tool in determining a safer, more simplistic plan in ERM surgery. If ILM peeling is the preferred strategy following ERM removal, this may be limited to patients showing focal ( $<50 \%)$ ERM-macula adhesion on preoperative SD-OCT avoiding unnecessary trauma to the retina.

\section{Summary}

\section{What was known before}

- Simultaneous inadvertent ILM peeling is a frequent occurrence in epiretinal membrane surgery.

\section{What this study adds}

- Simultaneous ILM peeling is frequent when there is a complete or broad ERM adhesion on preoperative SDOCT. The extent of simultaneous peeling is significantly dependent on the type of ERM adhesion to the inner retina. The extent of ERM-inner retina adhesion represents a valid predictor of simultaneous ILM peeling.

\section{Conflict of interest}

The authors declare no conflict of interest.

\section{References}

1 Machemer R. The surgical removal of epiretinal macular membranes (macular puckers) (author's transl). Klin Monatsbl Augenheilkd 1978; 173(1): 36-42.

2 Wong JG, Sachdev N, Beaumont PE, Chang AA. Visual outcomes following vitrectomy and peeling of epiretinal membrane. Clin Exp Ophthalmol 2005; 33(4): 373-378.

3 Dawson S, Shunmugam M, Williamson T. Visual acuity outcomes following surgery for idiopathic epiretinal membrane: an analysis of data from 2001 to 2011. Eye 2014; 28(2): 219-224.

4 Song SJ, Kuriyan AE, Smiddy WE. Results and prognostic factors for visual improvement after pars plana vitrectomy for idiopathic epiretinal membrane. Retina 2015; 35(5): 866-872.

5 Hisatomi T, Enaida H, Sakamoto T, Kagimoto T, Ueno A, Nakamura T et al. A new method for comprehensive bird'seye analysis of the surgically excised internal limiting membrane. Am J Ophthalmol 2005; 139(6): 1121-1132.

6 Park DW, Dugel PU, Garda J, Sipperley JO, Thach A, Sneed SR et al. Macular pucker removal with and without internal limiting membrane peeling: pilot study. Ophthalmology 2003; 110(1): 62-64.

7 Bovey EH, Uffer S, Achache F. Surgery for epimacular membrane: impact of retinal internal limiting membrane removal on functional outcome. Retina 2004; 24(5): 728-735.

8 Kwok AKh Lai TY, Yuen KS. Epiretinal membrane surgery with or without internal limiting membrane peeling. Clin Exp Ophthalmol 2005; 33(4): 379-385.

9 Oh HN, Lee JE, Kim HW, Yun IH. Clinical outcomes of double staining and additional ILM peeling during ERM surgery. Korean J Ophthalmol 2013; 27(4): 256-260.

10 Chang S, Gregory-Roberts EM, Park S, Laud K, Smith SD, Hoang QV. Double peeling during vitrectomy for macular pucker: the Charles L. Schepens lecture. JAMA Ophthalmol 2013; 131(4): 525-530.

11 Sandali O, El Sanharawi M, Basli E, Bonnel S, Lecuen N, Barale P-O et al. Epiretinal membrane recurrence: incidence, characteristics, evolution, and preventive and risk factors. Retina 2013; 33(10): 2032-2038.

12 Bu S-C, Kuijer R, Li X-R, Hooymans JM, Los LI. Idiopathic epiretinal membrane. Retina 2014; 34(12): 2317-2335.

13 Ripandelli G, Scarinci F, Piaggi P, Guidi G, Pileri M, Cupo G et al. Macular pucker: to peel or not to peel the internal limiting membrane? a microperimetric response. Retina 2014; 35(3): 498-507.

14 Ahn SJ, Ahn J, Woo SJ, Park KH. Photoreceptor change and visual outcome after idiopathic epiretinal membrane removal with or without additional internal limiting membrane peeling. Retina 2014; 34(1): 172-181.

15 Almony A, Nudleman E, Shah GK, Blinder KJ, Eliott DB, Mittra RA et al. Techniques, rationale, and outcomes of internal limiting membrane peeling. Retina 2012; 32(5): 877-891.

16 Carpentier C, Zanolli M, Wu L, Sepulveda G, Berrocal MH, Saravia $\mathrm{M}$ et al. Residual internal limiting membrane after epiretinal membrane peeling: results of the Pan-American Collaborative Retina Study Group. Retina 2013; 33(10): 2026-2031.

17 Wilkins JR, Puliafito CA, Hee MR, Duker JS, Reichel E, Coker JG et al. Characterization of epiretinal membranes using optical coherence tomography. Ophthalmology 1996; 103(12): 2142-2151. 
18 Tari SR, Vidne-Hay O, Greenstein VC, Barile GR, Hood DC, Chang S. Functional and structural measurements for the assessment of internal limiting membrane peeling in idiopathic macular pucker. Retina 2007; 27(5): 567-572.

19 Kinoshita T, Kovacs KD, Wagley S, Arroyo JG. Morphologic differences in epiretinal membranes on ocular coherence tomography as a predictive factor for surgical outcome. Retina 2011; 31(8): 1692-1698.

20 Mirza RG, Johnson MW, Jampol LM. Optical coherence tomography use in evaluation of the vitreoretinal interface: a review. Surv Ophthalmol 2007; 52(4): 397-421.

21 Falkner-Radler CI, Glittenberg C, Hagen S, Benesch T, Binder S. Spectral-domain optical coherence tomography for monitoring epiretinal membrane surgery. Ophthalmology 2010; 117(4): 798-805.

22 Koizumi H, Spaide RF, Fisher YL, Freund KB, Klancnik JM, Yannuzzi LA. Three-dimensional evaluation of vitreomacular traction and epiretinal membrane using spectral-domain optical coherence tomography. Am J Ophthalmol 2008; 145(3): 509-517.

23 Kim JS, Chhablani J, Chan CK, Cheng L, Kozak I, Hartmann K, Freeman WR. Retinal adherence and fibrillary surface changes correlate with surgical difficulty of epiretinal membrane removal. Am J Ophthalmol 2012; 153(4): 692-697.

24 Pavlidis M, Georgalas I, Körber N. Determination of a new parameter, elevated epiretinal membrane, by en face oct as a prognostic factor for pars plana vitrectomy and safer epiretinal membrane peeling. J Ophthalmol 2015; 2015: 838646.

25 Vedantham V. Indocyanine green-assisted internal limiting membrane removal in epiretinal membrane surgery. Am J Ophthalmol 2005; 139: 389.

26 Gaudric A, Fardeau C, Coberville M, Cohen D, Paques M, Mikol J. Ablation of the internal limiting membrane, macular unfolding and visual outcome in surgery of idiopathic epiretinal membranes. J Fr Ophthalmol 1993; 16: 571-576.

27 Shimada H, Nakashizuka H, Hattori T, Mori R, Mizutani Y, Yuzawa M. Double staining with brilliant blue G and double peeling for epiretinal membranes. Ophthalmology 2009; 116: 1370-1376.

28 Mori K, Gehlbach PL, Sano A, Deguchi T, Yoneya S. Comparison of epiretinal membranes in differing pathogenesis using optical coherence tomography. Retina 2004; 24(1): 57-62.

29 Pournaras CJ, Emarah A, Petropoulos IK. Idiopathic macular epiretinal membrane surgery and ILM peeling: anatomical and functional outcomes. Semin Ophthalmol 2011; 26: 42-46.

30 Clark A, Balducci N, Pichi F, Veronese C, Morara M, Torrazza $C$ et al. Swelling of the arcuate nerve fiber layer after internal limiting membrane peeling. Retina 2012; 32: 1608-1613.

31 Tadayoni R, Paques M, Massin P, Mouki-Benani S, Mikol J, Gaudric A. Dissociated optic nerve fiber layer appearance of the fundus after idiopathic epiretinal membrane removal. Ophthalmology 2001; 108: 2279-2283.

32 Ito Y, Terasaki H, Takahashi A, Yamakoshi T, Kondo M, Nakamura M. Dissociated optic nerve fiber layer appearance after internal limiting membrane peeling for idiopathic macular holes. Ophthalmology 2005; 112: 1415-1420.

33 Mitamura Y, Suzuki T, Kinoshita T, Miyano N, Tashimo A, Ohtsuka K. Optical coherence tomographic findings of dissociated optic nerve fiber layer appearance. Am J Ophthalmol 2004; 137: 1155-1156.

34 Imai H, Ohta K. Microperimetric determination of retinal sensitivity in areas of dissociated optic nerve fiber layer following internal limiting membrane peeling. Jpn J Ophthalmol 2010; 54: 435-440.

35 Steven P, Laqua H, Wong D, Hoerauf H. Secondary paracentral retinal holes following internal limiting membrane removal. Br J Ophthalmol 2006; 90(3): 293-295.

36 Falkner-Radler CI, Glittenberg C, Gabriel M, Binder S. Intrasurgical microscope- integrated spectral domain optical coherence tomography-assisted membrane peeling. Retina 2015; 35(10): 2100-2106. 